\title{
Ileocolic mucormycosis - an unusual cause of a mass in the right iliac fossa
}

\author{
Kishanchand Chethan MS, Seetharam Prasad MS DNB MRCSEd, Lingadakai Ramachandra MS
}

\begin{abstract}
K Chethan, S Prasad, L Ramachandra. Ileocolic mucormycosis - an unusual cause of a mass in the right iliac fossa. Can J Infect Dis Med Microbiol 2012;23(3):e65-66.
\end{abstract}

Mucormycosis is a relatively uncommon, aggressive and lethal mycosis. Fungi from the order Mucorales are the etiological agents of mucormycosis. The condition is more common among the immunocompromised, diabetic patients with ketoacidosis and people with iron overload syndromes. Diagnosis of mucormycosis requires a high index of suspicion regarding the possibility of the condition in high-risk individuals. Timely diagnosis is critical to survival and minimization of morbidity. A favourable outcome is possible only if appropriate treatment is initiated as early as possible. The present article reports a case of ileocolic mucormycosis involving a patient with chronic renal failure and familial hyperuricemia.

Key Words: Amphotericin B; Immunocompromised; Mucormycosis; Zygomycosis

$\mathrm{M}$

ucormycosis is a rare, opportunistic fungal infection seen among immunocompromised patients. Gastrointestinal (GI) mucormycosis involving the ileocolic region is extremely rare. A case of ileocolic mucormycosis can pose significant challenge to the treating surgeon both for diagnosis and management. We present a case of ileocolic mucormycosis that was successfully managed nonoperatively, as well as a brief review of the relevant literature.

\section{CASE PRESENTATION}

A 31-year-old male farmer presented with complaints of abdominal pain, fever and passage of black-coloured stools. The patient had a history of chronic renal failure with familial hyperuricemia and hypertension. With regular hemodialysis, his serum creatinine level was maintained at approximately $442 \mathrm{mmol} / \mathrm{L}$. His nutritional status was adequate and there were no comorbidities, including diabetes and HIV infection, which would render him immunocompromised. There was no history of administration of steroids in the recent past. Abdominal examination revealed a tender, irregular mass measuring $6 \mathrm{~cm} \times 8 \mathrm{~cm}$ in the right iliac fossa. Laboratory investigations revealed the following data: hemoglobin level of $93 \mathrm{~g} / \mathrm{L}$, total leukocyte count of $4.16 \times 10^{9} / \mathrm{L}$, blood urea nitrogen concentration of $15,470 \mathrm{mmol} / \mathrm{L}$, serum creatinine concentration of $680 \mathrm{mmol} / \mathrm{L}$ and serum potassium concentration of $6 \mathrm{mmol} / \mathrm{L}$. An arterial blood gas analysis yielded values suggestive of metabolic acidosis. An ultrasound scan of the abdomen and pelvis found multiple, thickened, dilated, fluid-filled, hyperperistaltic bowel loops and shrunken kidneys with increased cortical echogenecity. A provisional diagnosis of ileocecal tuberculosis or an appendicular mass was made. Hemodialysis was performed to stabilize the deteriorating renal parameters. Diagnostic esophagogastroscopy and colonoscopy were performed. While the esophagogastroscopy was normal, colonoscopy revealed multiple ulcerative lesions in the cecum with edematous cecal mucosa (Figure 1). Ulcers were also seen in the terminal ileum. Blood culture and serological tests for Salmonella typhi were negative. Histopathological examination of the biopsies taken from the ileum and the cecum showed

\section{La mucormycose iléocolique : une cause inhabituelle de masse dans la fosse iliaque droite}

La mucormycose est une mycose relativement rare, agressive et fatale. Les champignons de l'ordre Mucorales en sont les agents étiologiques. La pathologie est plus courante chez les patients diabétiques immunosupprimés ayant une acidocétose et chez les personnes ayant des syndromes de surcharge en fer. Pour poser un diagnostic de mucormycose, il faut un fort indice de suspicion quant à sa possibilité chez les personnes très vulnérables. Il est essentiel de poser le diagnostic rapidement pour assurer la survie et réduire au minimum la morbidité. Si on veut favoriser une issue positive, il faut absolument amorcer un traitement convenable le plus tôt possible. Le présent article traite d'un cas de mucormycose iléocolique touchant un patient atteint d'insuffisance rénale chronique et d'hyperuricémie familiale.

Department of Surgery, Kasturba Medical College, Manipal, India

Correspondence: Dr Seetharam Prasad, Department of Surgery, Kasturba Medical College, Manipal, Karnataka, India.

Telephone 0953-8270192, fax 0820-2570061, e-mail drprasadseetharam@yahoo.co.in 
mucormycosis and zygomycosis are used synonymously, the former term would appear more appropriate because the class Zygomycetes also includes the order Entomophthorales, as well as the order Mucorales (1). The fungus exists in two forms, the infective sporangiospores and the hyphal form responsible for tissue necrosis and dissemination. The pathogenicity of the fungus is due to direct tissue penetration and angioinvasion resulting in vascular thrombosis and tissue necrosis (1). Depending on the site of involvement, mucormycosis can be classified as rhinocerebral, pulmonary, cutaneous, GI, central nervous system or disseminated mucormycosis.

GI mucormycosis is the rarest of all forms of mucormycosis, accounting for approximately $7 \%$ of all cases (2). GI mucormycosis most commonly involves the stomach $(57.5 \%)$, followed by the colon $(32.3 \%)$ and the ileum $(6.9 \%)$. Fewer than 20 cases of GI mucormycosis with colonic involvement have been reported in the past two decades (3). GI infection most probably results from ingestion of fungal spores. Being an opportunistic infection, some degree of immunocompromised status is almost always associated with GI mucormycosis. The most common underlying risk factors for mucormycosis include poorly controlled diabetes mellitus, administration of high-dose, systemic corticosteroids in solid organ and hematopoietic stem cell transplantation, penetrating trauma or burns, persistent neutropenia and deferoxamine-based therapy (4). Chronic renal failure has not been described in the literature as a traditional risk factor for mucormycosis. Although chronic renal failure has not been described as a traditional risk factor for mucormycosis, a study from India (5) observes a significant association of chronic renal failure among patients with pulmonary mucormycosis. GI mucormycosis usually presents with nonspecific symptoms, such as abdominal pain, abdominal distension, fever and diarrhea. Patients can also present with intestinal perforation, obstruction or hemorrhage (2). Even though melena is a feature of upper GI bleeding, because we failed to demonstrate any specific lesion causing an upper GI bleed, it was presumed that ileocecal mucormycosis was responsible for melena in this patient. Lack of pathognomonic clinical features renders early diagnosis of GI mucormycosis very difficult. Although a vigilant clinician can suspect the possibility of GI mucormycosis in a patient with traditional risk factors, the same cannot be expected in a patient without traditional risk factors. Ileocolic mucormycosis presenting with abdominal pain, fever and a mass in the right iliac fossa is extremely difficult to differentiate from other, similarly presenting, more common clinical conditions, such as an appendicular mass or ileocecal tuberculosis. A definitive diagnosis of mucormycosis is almost always ascertained by histopathological evidence of fungal invasion of tissue. Colonoscopic studies may help in diagnosis of ileocolic mucormycosis in some cases. Endoscopic features of ileocolic mucormycosis include hemorrhagic edematous mucosa with erosions, dark ulcers and, rarely, a mushroom-like fungal mass, which is

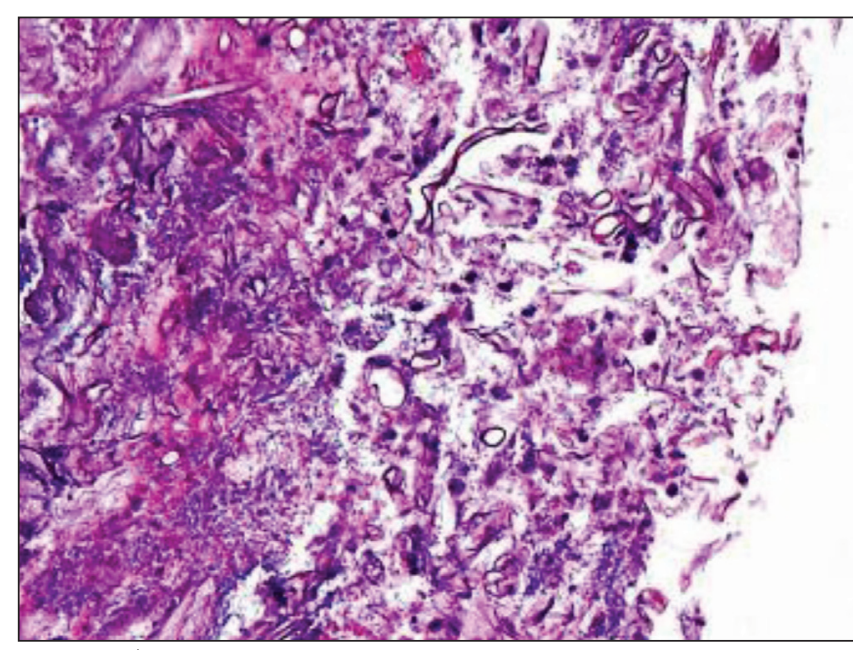

Figure 2) Microscopy showing hyphae

occasionally covered by a black crust (3). There have been no prospective studies evaluating the primary treatment of mucormycosis and, as a result, there is uncertainty regarding the optimal treatment of the condition. Recommended treatments of ileocolic mucormycosis include antifungal therapy, surgery or a combination of both. We did offer surgical resection to the patient, but the patient was not willing to undergo any surgical procedure. Amphotericin B at the highest tolerated dose is the most commonly used antifungal treatment for mucormycosis. Surgery may be particularly useful in cases where infection remains localized, with the goal of removing as much of the infected or devitalized tissue as possible. For ileocolic mucormycosis, this may amount to bowel resections of varying extent.

\section{REFERENCES}

1. Kontoyiannis DP, Lewis RE. Invasive zygomycosis: Update and pathogenesis, clinical manifestation and management. Infect Dis Clin N Am 2006;20:581-607.

2. Thomson SR, Bade PG, Taams M, Chrystal V. Gastrointestinal mucormycosis. Br J Surg 1991;78:739-41.

3. Lo OS, Law WL. Ileocolonic mucormycosis in adult immunocompromized patients: A surgeons prospective. World J Gastroenterol 2010;16:1165-70.

4. Roden MM, Zaotis TE, Buchanan WL, et al. Epidemiology and outcome of zygomycosis: A review of 929 reported cases. Clin Infect Dis 2005;41:634-53.

5. Chakrabarti A, Chatterjee SS, Das A, et al. Invasive zygomycosis in India: Experience in a tertiary care hospital. Postgrad Med J 2009;85:573-81. 


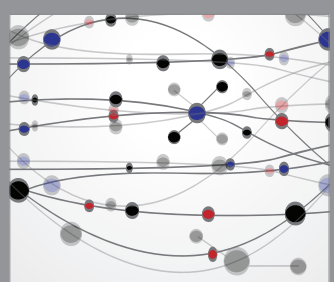

The Scientific World Journal
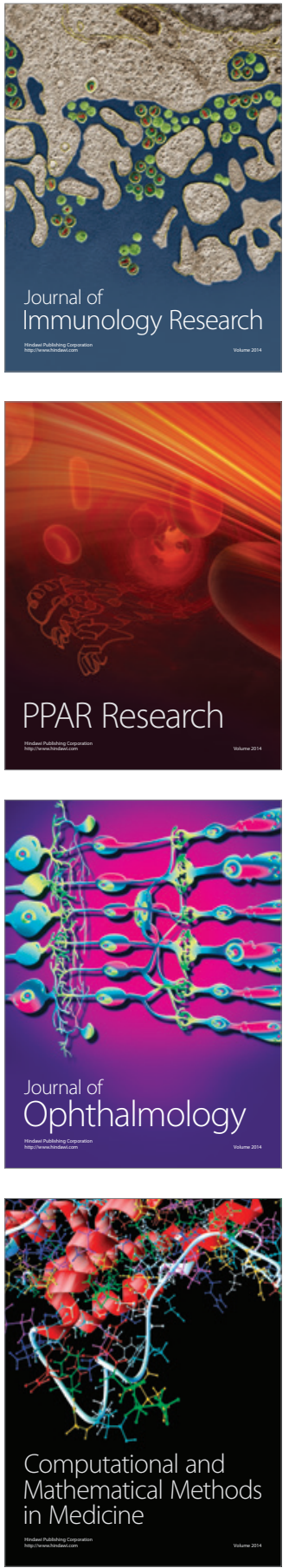

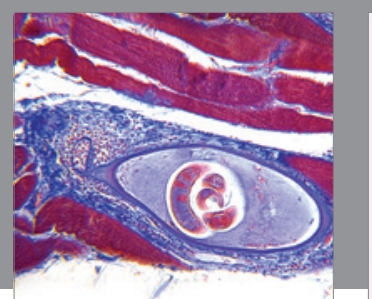

Gastroenterology Research and Practice

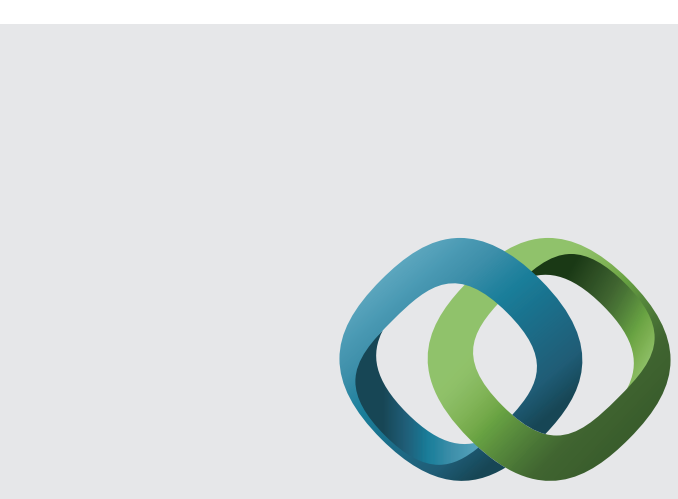

\section{Hindawi}

Submit your manuscripts at

http://www.hindawi.com
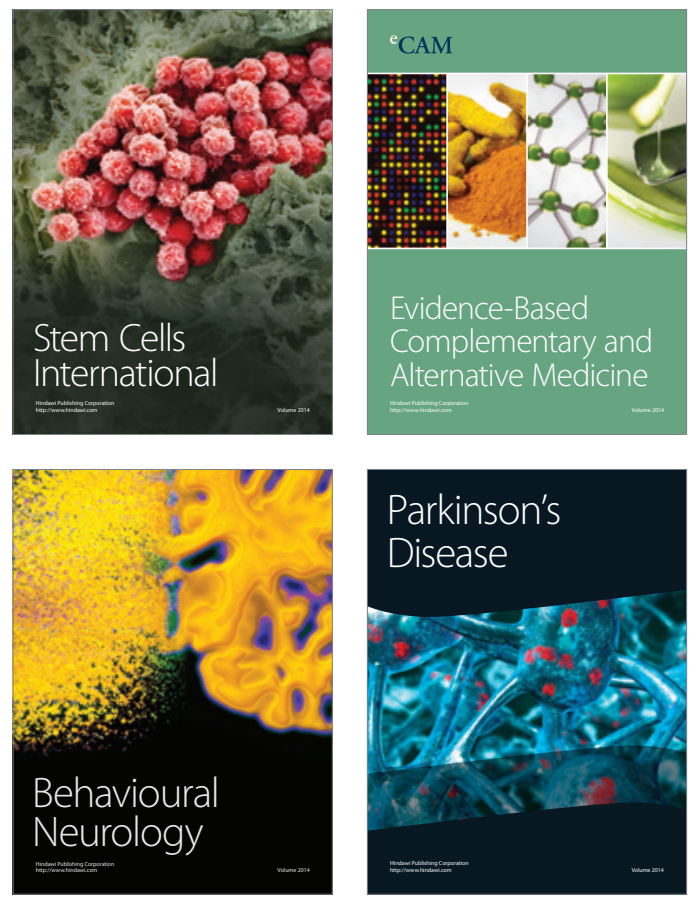
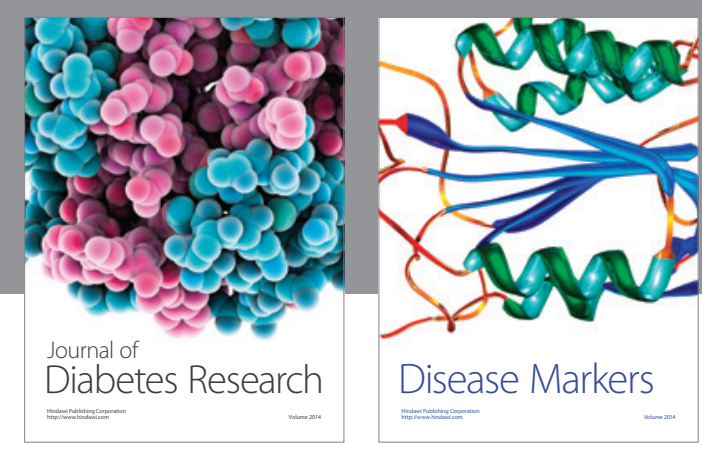

Disease Markers
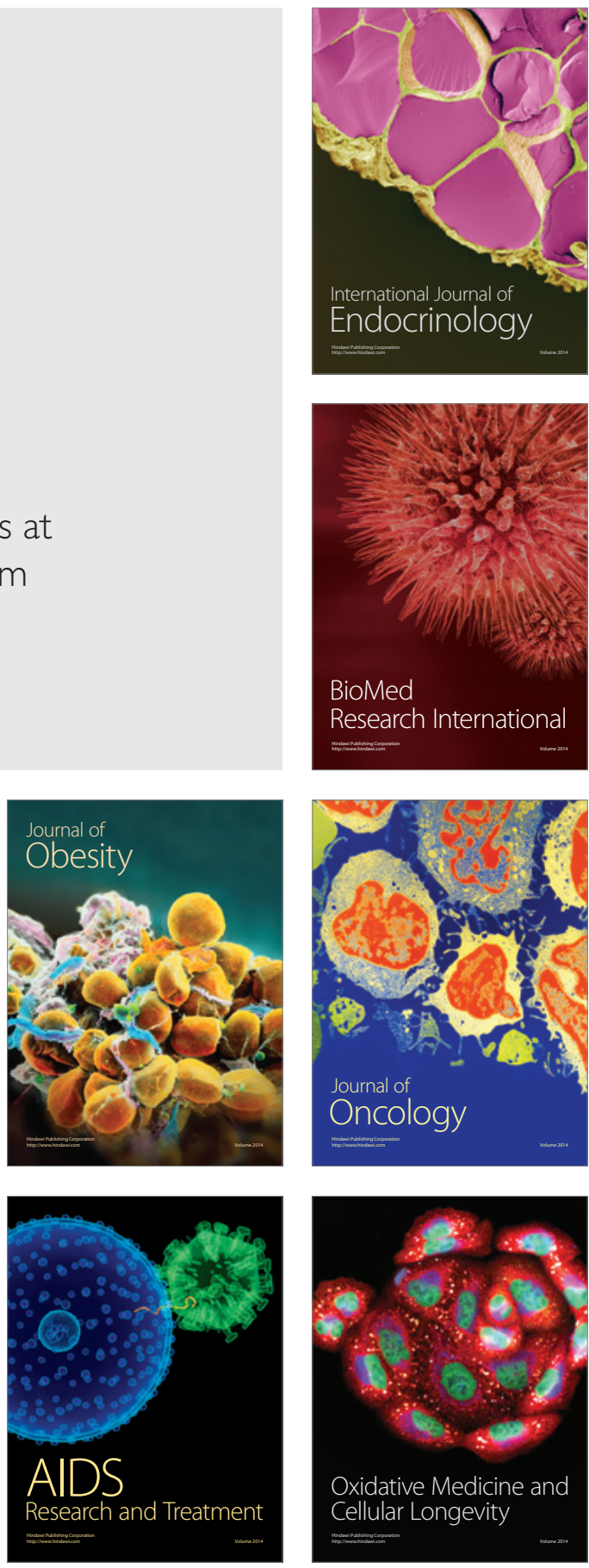